\title{
Transvaginal Adhesiolysis Using Laparoscopic Instruments
}

\author{
Qiuqi Angela Shan, MD, Jon K. Hathaway, MD, PhD
}

Department of Obstetrics and Gynecology, Indiana University School of Medicine, Indianapolis, Indiana (both authors).

\begin{abstract}
Intra-abdominal adhesions are a common complication of abdominal surgery and can lead to further complications including bowel obstruction, infertility, and pain. We describe a novel technique of performing transvaginal laparoscopic lysis of adhesions following a total vaginal hysterectomy in a 41-year-old woman with periumbilical pain and menorrhagia. This natural orifice transluminal endoscopic surgical procedure was tolerated well, did not add considerable overall operating time, and did not require extensive additional training. The patient reported no periumbilical pain at 1 year.
\end{abstract}

Key Words: Surgery-induced tissue adhesions, Laparoscopic surgery, Natural orifice transluminal endoscopic surgery, Hysterectomy, Vaginal

Citation Shan QA, Hathaway JK. Transvaginal adhesiolysis using laparoscopic instruments. CRSLS e2014.00220. DOI: 10.4293/CRSLS.2014.00220.

Copyright (C) 2014 SLS This is an open-access article distributed under the terms of the Creative Commons Attribution-Noncommercial-ShareAlike 3.0 Unported license, which permits unrestricted noncommercial use, distribution, and reproduction in any medium, provided the original author and source are credited.

Address correspondence to: Jon K. Hathaway, Department of Obstetrics and Gynecology, Indiana University School of Medicine, 550 North University Boulevard, Room 2440, Indianapolis, IN 46202. Telephone: (317) 630-8426, Fax: (317) 630-6524, E-mail: jkhathaw@iupui.edu

\section{INTRODUCTION}

Intra-abdominal adhesions commonly occur after open abdominal or pelvic surgery. ${ }^{1,2}$ Adhesions themselves can lead to complications, from small bowel obstruction ${ }^{2}$ to infertility to chronic pain. ${ }^{1}$ The presence of adhesions complicates future surgical procedures, especially for the initial port, which is often placed with minimal visualization. The most minimally invasive surgery uses the complete natural orifice transluminal endoscopic surgery (NOTES) approach. ${ }^{3-6}$ In this respect, gynecologic surgeons have been at the forefront of the field, performing purely transvaginal surgeries such as total vaginal hysterectomies (TVHs) and pelvic floor repairs long before NOTES was used for other intra-abdominal procedures. ${ }^{7}$ As such, we stand in an ideal position to pioneer new techniques for performing intra-abdominal or pelvic procedures via the vaginal orifice.

The following novel NOTES case is a report of laparoscopic lysis of adhesions via transvaginal route following a TVH.

\section{Case}

The patient was a 41-year-old Gravida 6, para 4, white female with debilitating menorrhagia for 2 years. She complained of heavy and irregular menses that often lasted for 2 weeks. She also described constant abdominal pain in the pelvic to umbilicus area that did not change with menstruation or bowel function. An ultrasound revealed a small $(6 \mathrm{~mm} \times 6 \mathrm{~mm} \times 8 \mathrm{~mm})$ anterior fundal serosal fibroid. After extensive discussion of risks and benefits of various treatment options, the patient declined medical management or ablation and desired definitive management with hysterectomy. The surgical approach to examining the intra-abdominal cavity for a possible source of her pain was complicated by her history of gunshot wounds to the abdomen at the age of 12 , for which she received exploratory laparotomy through a midline vertical incision.

Based on her physical examination findings and our vaginal surgery experience, she was a good candidate for TVH, and she was consented for possible transvaginal laparoscopy after the removal of her uterus. The patient underwent TVH and a 182-g benign-appearing uterus was removed without complications. The abdominal cavity was visualized using a SILS port (Covidien, Dublin, Ireland) inserted through the open cuff and a $30^{\circ}$ angle scope. Multiple omental adhesions were seen to the periumbilical region and the midline vertical incision of the patient's previous exploratory 
Transvaginal Adhesiolysis Using Laparoscopic Instruments, Shan and Hathaway.

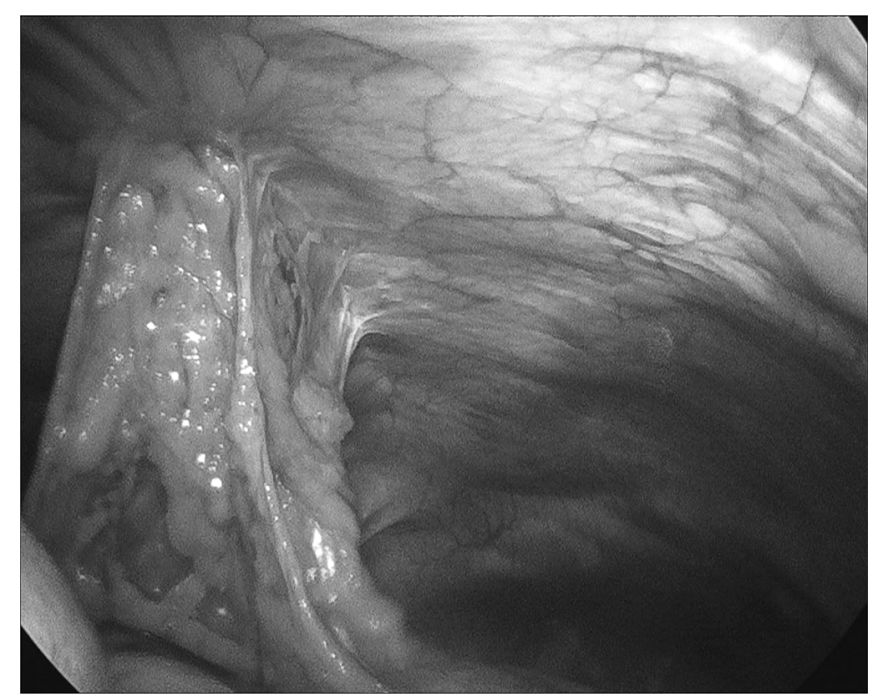

Figure 1. Periumbilical omental adhesions as seen using vaginal laparoscopy.

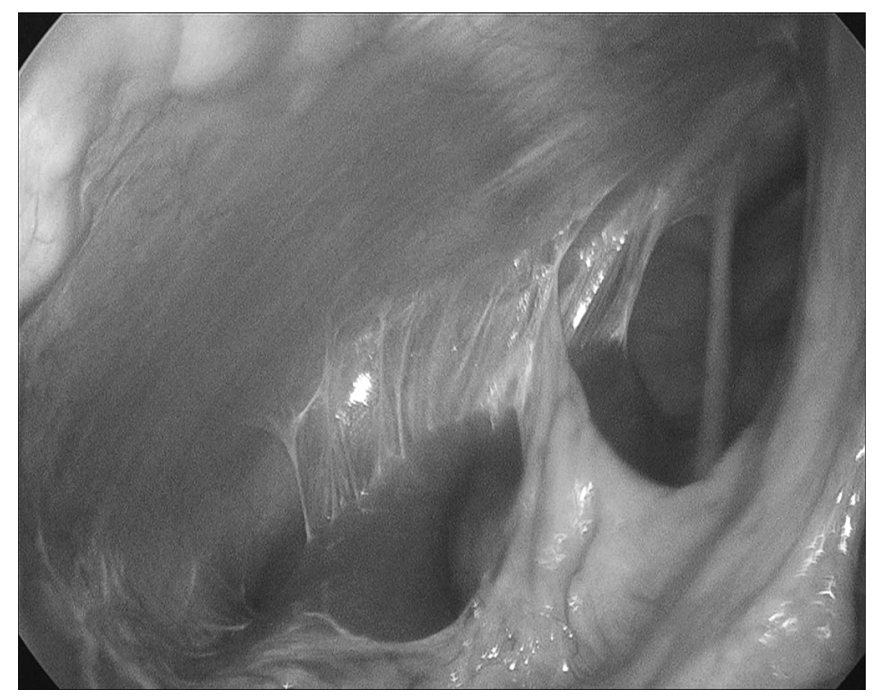

Figure 2. Perihepatic omental adhesions.

laparotomy (Figures 1 and 2). No other abnormalities were visualized. A Harmonic Ace scalpel device (Ethicon, Cincinnati, Ohio) was inserted through the SILS port and lysis of all accessible adhesions was performed. A total of 15 minutes was spent on this part of the surgery (Figures 3 and 4). No bowel was present in the adhesions and the adjacent bowel appeared normal. Hemostasis was ensured and the vaginal cuff was closed in the vertical fashion. There was no evidence of retroperitoneal emphysema.

The patient recovered well and her postoperative course was uncomplicated. At 1-year follow-up, she is doing well and without periumbilical pain.

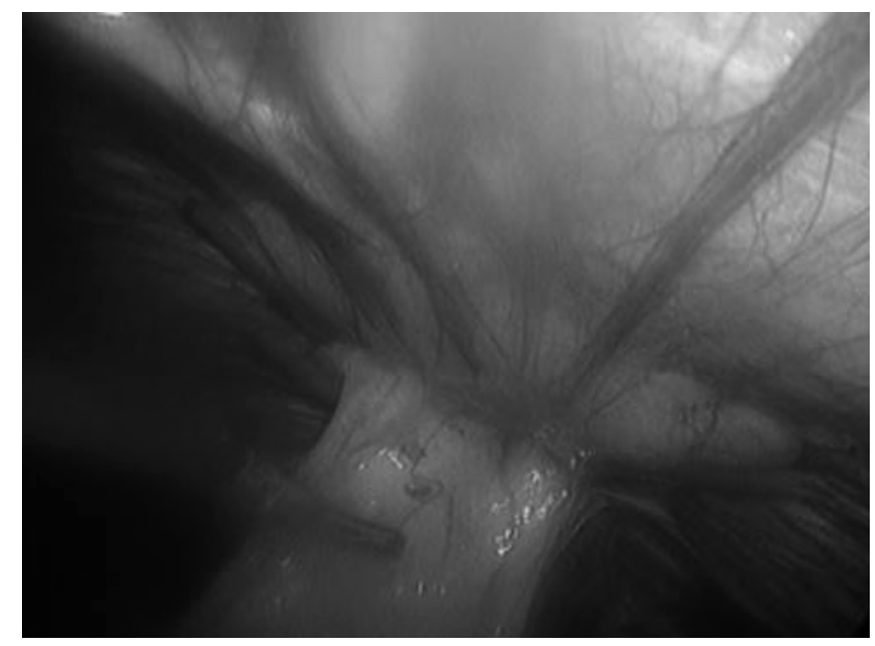

Figure 3. Taking down adhesions using Harmonic Ace scalpel.

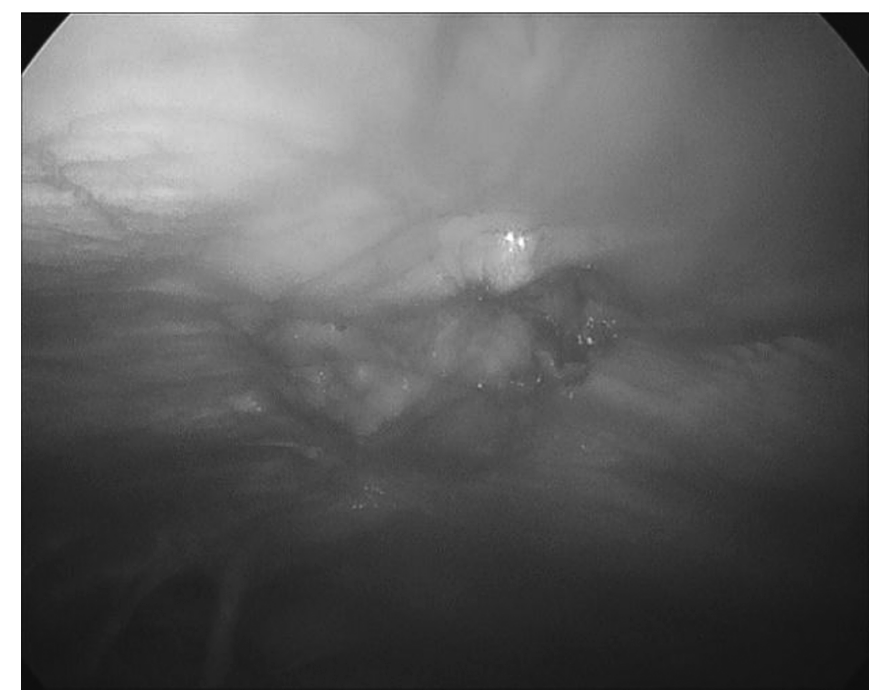

Figure 4. Postadhesiolysis of periumbilical adhesions.

\section{DISCUSSION}

Adhesion formation occurs in $\geq 90 \%$ of patients who undergo abdominal or pelvic surgeries. ${ }^{1}$ Complications of adhesions can be life-threatening, such as bowel obstruction. ${ }^{1,2}$ In women, $15 \%$ to $40 \%$ of infertility cases have been attributed to adhesions. ${ }^{1}$ Chronic pelvic pain is frequently attributed to adhesions, although this relationship is questionable and unclear. Regardless, adhesion formation is an unwanted outcome of abdominal and pelvic surgery. ${ }^{1,8}$

Despite the lack of beneficial evidence, lysis of adhesions is commonly performed. In 1994, over 300,000 hospitalizations in the United States were directly attributed to adhesiolysis procedures, $47 \%$ of which followed gynecologic surgeries. ${ }^{9}$ 
Reportedly, 1 of 22 readmissions following open surgery of the reproductive tract are directly related to adhesions. ${ }^{10}$

Adhesiolysis has traditionally been performed either via open surgery or abdominal laparoscopy. ${ }^{11}$ It can be argued that these methods are counterproductive, as the procedure itself increases the risk of the complication it was attempting to correct. We postulate that adhesiolysis can be safely and effectively performed via a purely transvaginal route, especially with concomitant vaginal hysterectomy. Currently available laparoscopic instrumentation allows for adequate exploration of the abdomen and pelvis via the transvaginal route.

This approach follows the credo of NOTES to gain access to intra-abdominal and pelvic pathology. 3,4,6 A few cases have been reported in the literature of using transvaginal NOTES for the evaluation of or removal of benign adnexal pathology, ${ }^{12}$ appendectomy, ${ }^{13}$ and nephrectomy. ${ }^{14}$ We could find no reported cases of laparoscopic adhesiolysis via the transvaginal route. We believe this is a novel approach that fulfills the need of performing lysis of adhesions and exploration of the abdomen and pelvis without a concomitant increased risk of adhesion formation or trocar injury.

The three challenges we faced were the fixed angle of the scope requiring us to operate at the edge of the visual field, the length of the instruments were not optimal to pass from the vagina to beyond the umbilicus, and the persistent loss of pneumoperitoneum.

The surgery would have been easier if we had access to a flexible tip scope to allow dynamic angling of the lens for better visualization. We also did not have access to longer instruments such as those used in bariatric surgeries to access the more cephalic regions of the abdomen. Maintaining the pneumoperitoneum proved to be a challenge and, in the end, we used an Allis clamp distal to the SILS port to achieve adequate pneumoperitoneum. In retrospect, if we had performed a temporary purse-string suture around the middle of the SILS port, we might have had an easier time maintaining a pneumoperitoneum. Alternatively, a SILS-style port with an inflatable balloon might also have been useful, but one is not currently available. However, given the compliance of the vaginal wall, the engineering may prove to be difficult.

In this case report, the patient underwent transvaginal laparoscopic lysis of adhesions following TVH with improvement of her symptoms. The procedure was well tolerated and did not take significant additional operative time. Gynecologic surgeons are familiar with both laparoscopic and vaginal surgery and thus are uniquely qualified to combine these procedures with little or no additional training.

\section{References:}

1. De Wilde RL, Brölmann H, Koninckx PR, et al., for the Anti-Adhesions in Gynecology Expert Panel (ANGEL). Prevention of adhesions in gynaecological surgery: the 2012 European field guideline. Gynecol Surg. 2012;9(4):365-368.

2. Ellis H. The clinical significance of adhesions: focus on intestinal obstruction. Eur J Surg Suppl. 1997;(577):5-9.

3. Khashab MA, Kalloo AN. NOTES: current status and new horizons. Gastroenterology. 2012;142(4):704-710.e1.

4. Moris DN, Bramis KJ, Mantonakis EI, Papalampros EL, Petrou AS, Papalampros AE. Surgery via natural orifices in human beings: yesterday, today, tomorrow. Am J Surg 2012;204(1):93-102.

5. Nau P, Anderson J, Happel L, et al. Safe alternative transgastric peritoneal access in humans: NOTES. Surgery. 2011;149(1):147-152.

6. Pearl JP, Ponsky JL. Natural Orifice translumenal endoscopic surgery: a critical review. J Gastrointest Surg. 2008;12(7):1293-1300.

7. Moen MD, Noone MB, Elser DM, for the Urogynecology Network. Natural orifice hysterectomy. Int Urogynecol J Pelvic Floor Dysfunct. 2008;19(9):1189-1192.

8. Ten Broek RP, Kok-Krant N, Bakkum EA, Bleichrodt RP, van Goor H. Different surgical techniques to reduce post-operative adhesion formation: a systematic review and meta-analysis. Hum Reprod Update. 2013;19(1):12-25.

9. Ray NF, Denton WG, Thamer M, Henderson SC, Perry S. Abdominal adhesiolysis: inpatient care and expenditures in the United States in 1994. J Am Coll Surg. 1998;186(1):1-9.

10. Lower AM, Hawthorn RJ, Ellis H, O'Brien F, Buchan S, Crowe AM. The impact of adhesions on hospital readmissions over ten years after 8849 open gynaecological operations: an assessment from the Surgical and Clinical Adhesions Research Study. BJOG. 2000;107(7):855-862.

11. Grafen FC, Neuhaus V, Schöb, Turina M. Management of acute small bowel obstruction from intestinal adhesions: indications for laparoscopic surgery in a community teaching hospital. Langenbecks Arch Surg. 2010;395(1):57-63.

12. Ahn KH, Song JY, Kim SH, Lee KW, Kim T. Transvaginal single-port natural orifice transluminal endoscopic surgery for benign uterine adnexal pathologies. J Minim Invasive Gynecol. 2012;19(5):631-635.

13. Roberts KE, Solomon D, Mirensky T, et al. Pure transvaginal appendectomy versus traditional laparoscopic appendectomy for acute appendicitis: a prospective cohort study. Ann Surg. 2012;255(2):266-269.

14. Kaouk JH, Haber GP, Goel RK. Pure natural orifice translumenal endoscopic surgery (NOTES) transvaginal nephrectomy. Eur Urol. 2010;57(4):723-726. 